However, it cannot provide the Office for the Suppression of Fraud with the same conclusive evidence as that provided by the starch indicator and by an analysis of its chemical composition.

\title{
SUR LES DÉFAUTS DE L'APPLICATION PRATIQUE DE DIFFÉRENTES RÉGLEIMENTATIONS CONCERNANT LE CONTROLE DE LA COMPOSITION CHIMIQUE DE FROMAGES
}

\author{
par \\ Docteur Dj. FILLIPOVITCH \\ Service Vétérinaire Fédéral-Belgrade
}

\begin{abstract}
Parmi les denrées d'origine animale, les fromages présentent la plus grande diversité au point de vue de la production et de la composition chimique. Par conséquent, il est impossible d'adopter un standard uniforme pour les normes de constituants chimiques des fromages. Il existe à ce sujet beaucoup de publications, particulièrement dans les compte rendus des Congrès Internationaux sur le lait et ses dérivés et dans les divers bulletins de laiterie.

Dans les rapports très nombreux exposés au xIve Congrès International sur le lait et ses dérivés, tenu à Rome en 1956, on trouve les données très instructives sur la législation nationale de plusieurs pays et sur les conventions internationales concernant les fromages. Malheureusement, on peut constater dans l'ensemble de ces dispositions réglementaires certaines prescriptions assez compliquées pour l'application pratique. La situation est semblable presque partout dans les publications traitant ces problèmes, et en particulier sur la teneur des fromages en matière grasse.

Nous sommes pleinement d'accord avec W.A. Lethem [1] quand il dit que le premier principe pour toute loi est que celle-ci soit effectivement appliquée; le second étant qu'elle soit acceptée par la plupart des intéressés. Il est vrai qu'aucun système de contrôle ne peut être efficace sans l'assistance active de la plupart des intéressés, et sans l'appui d'une opinion publique éclairée. A mon avis, il ne faudrait pas croire que dans tous les cas et dans tous les pays l'éducation des fermiers et des commerçants, ainsi que tous de les autres intéressés, donnera de bons effets. Pour certains cas, il faut imposer des mesures législatives très sévères pour protéger le consommateur en premier lieu, soit lors de la vente dans le pays de production, soit dans les cas d'exportation. G. Thieulin [2], dans son étude critique de la législation française concernant le lait, considère que, malgré la base valable que consti-
\end{abstract}


tuent ces dispositions réglementaires, elles sont plus répressives que préventives, et, dans ces conditions, ne permettent que des interventions tardives sinon, souvent, illusoires.

Pour voir combien nous sommes toujours loin de trouver une plus grande souplesse du point de vue de la classification des fromages selon leur teneur en matière grasse dans l'extrait sec, nous allons exposer la législation à ce sujet, seulement pour exemple, de quelques pays. Il est inutile de revenir sur ce fait que le dosage de la matière grasse dans le fromage présente le plus important travail dans le but du contrôle de la composition chimique. Bien entendu, il est impossible de se borner uniquement sur la détermination de la matière grasse dans le fromage car elle se calcule par rapport à la matière sèche, et, par conséquent, on fait également le dosage de l'extrait sec. De telle façon on obtient le taux de matière grasse et d'humidité.

En Yougoslavie, le règlement sur la qualité des denrées alimentaires, des conditions pour leur production et la vente, du 20 mars 1957 [3], prévoit la classification suivante des fromages selon leur teneur en matière grasse dans l'extrait sec :

1. "Extra gras", contenant au minimum $55 \%$ de matière grasse et maximum $40 \%$ d'humidité ;

2. "Totalement gras ", contenant au minimum $45 \%$ de matière grasse ;

3. "Trois-quart gras", au minimum $35 \%$ de matière grasse ;

4. "Demi gras ", au minimum $25 \%$ de matière grasse ;

5. "Un quart gras", au minimum $15 \%$ de matière grasse ;

6. "Maigre ", au-dessous de $15 \%$ de matière grasse.

Le fromage frais ne devra jamais contenir plus de $75 \%$ d'humidité.

Les fromages frais à pâte molle préparés avec du lait de vache ou de brebis, ou bien avec du mélange de ces deux laits, sous la dénomination "fromage blanc en tranches", devra renfermer au minimum $45 \%$ de matière grasse et ne devra pas renfermer plus de $65 \%$ d'humidité.

Les fromages " demi gras » de l'espèce précédente doivent contenir au minimum $25 \%$ de matière grasse, dans l'extrait sec.

Comme on le voit bien nous avons six variétés de fromages fermes et deux sortes de "fromages blanes en tranches". Ajoutons que le fromage "fondu " doit contenir au minimum $40 \%$ de matière grasse, tandis que le fromage «fondu demi gras », doit contenir au minimum $25 \%$ de matière grasse, toujours dans l'extrait sec.

Il est prévu que tous les fromages en vente doivent porter 
la désignation de l'espèce et la teneur en matière grasse calculée dans la matière sèche. Les fromages mis en vente sans une telle désignation doivent remplir les conditions précédentes au point de vue de la qualité chimique ; c'est-à-dire du taux de matière grasse.

D'après A. Rochaix et A. TAPERnoux [4] la plupazt des fromages français, selon la législation en vigueur, doivent avoir au minimum 40 grammes de matière grasse pour 100 grammes de fromage après complète dessication.

En Bulgarie, d'après le Recueil du Standard bulgare pour le lait et ses produits (Sofia, 1956) [5], la classification des fromages est très simple, ce qui est valable uniquement pour le fromage de ferme "KASKAVALJ " pour lequel on prévoit :

- minimum pour le fromage "totalement gras" : $46 \%$ de ma tière grasse ;

- minimum pour le fromage "demi gras » : $28 \%$ de matière grasse dans l'extrait sec.

En Norvège, selon Gunnar Aas [6] il existe le standard suivant pour la teneur des fromages en matière grasse dans' l'extrait sec :

1. "Totalement gras" au minimum : $45 \%$ :

2. "Demi gras ", au minimum : $30 \%$;

3. "Quart gras ", au minimum : $20 \%$;

4. "Maigre", au-dessous de : $20 \%$.

En Suède, d'après Erik CARLBERG [7], la classification selon la teneur des fromages en matière grasse dans l'extrait sec est la suivante :

1. "Totalement gras", au minimum : $45 \%$;

2. "Trois quart gras", au minimum : $40 \%$;

3. "demi gras", au minimum $30 \%$;

4. "Un quart gras", au minimum $20 \%$;

5. "Maigre ", au-dessous de : $20 \%$.

La législation allemande, d'après Georg RoEDER [8], prévoit huit variétés de fromages selon leur teneur en matière grasse dans l'extrait sec, à savoir :

1. "Double crème ", au minimum : $60 \%$;

2. "crème fromage ", au minimum : $50 \%$;

3. "Totalement gras », au minimum : 45\%";

4. "gras ", au minimum : $40 \%$;

5. "Trois quart gras", au minimum : $30 \%$;

6. "Demi gras ", au minimum : $20 \%$;

7. "Un quart gras", au minimum $10 \%$;

8. "Maigre", au-dessous de $10 \%$. 
En raison de la place limitée, nous nous sommes borné à eiter seulement ces quelques exemples de divers pays. L'adoption d'un standard uniforme pour la composition chimique de fromage, en particulier sur les éléments les plus importants de fromage : matière grasse, extrait sec et humidité, serait très utile. Cependant, on rencontre de grandes difficultés pour exiger l'adoption de standards nternationaux en général, car il faut tenir compte de la différence énorme de l'évolution de la production, du commerce et du goût, très différents, des consommateurs dans les divers pays.

Comme il ne faut pas chercher à comparer des choses incomparables, il est illusoire d'essayer de fixer les mêmes textes législatifs relatifs aux conditions de fabrication des fromages, par exemple, en France, qui est le pays le plus connu dans le monde par larichesse en variété de toutes sortes de fromages, et dans un pays où on fabrique seulement quelques espèces de ces produits laitiers. Cependant, dans la nouvelle législation de certains pays concernant la teneur des fromages en matière grasse, on trouve les mêmes normes légales que dans un autre pays où les conditions de production, de vente et de contrôle sont tout à fait différentes.

Les défauts d'application pratique de différentes réglementations se manifestent particulièrement dans les cas où la production de fromage est répartie sur un grand nombre de petits producteurs qui sont en même temps les vendeurs directs sur les marchés dispersés partout dans les pays considérés. On sait très bien que les consommateurs éprouvent de grandes difficultés pour apprécier les qualités et la valeur du fromage mis en vente. D'autre part, les petits producteurs de fromage n'ont pas la possibilité d'examiner leurs fromages au point de vue de la composition chimique, même limitée au dosage de la matière grasse.

Par conséquent, nos paysans qui représentent la majorité de nos producteurs et vendeurs de fromages aussi bien à pâte molle qu'à pâte ferme, se bornent à vendre presque une seule sorte de fromage, sous la simple dénomination "fromage", malgré les prescriptions en vigueur depuis 1957 selon lesquelles nous avons en Yougoslavie six variétés de fromages d'après leur teneur en matière grasse dans l'extrait sec, avec encore quelques autres variétés non groupées.

Dans notre pays, nous n'avons jamais produit le fromage "extra gras» et à l'heure actuelle, il en existè seulement sur le papier dans le réglement déjà cité. On peut dire la même chose pour les fromages " trois quart gras ", " demi gras " et " quart gras". En réalité, nous avons, au marché, les fromages à pâte molle et ferme vendus sous la dénomination unique "totalement gras", ayant un différent pourcentage d'humidité. 
En ce qui concerne la limite de la teneur en eau, il est tout à fait discutable. Faut-il la prescrire ou non pour plusieurs raisons pratiques? Pour la plupart des consommateurs, ce n'est pas une question de grande importance que certains fromages contiennent un peu plus d'humidité à la condition qu'ils correspondent à la qualité organoleptique et en particulier qu'ils soient préparés avec du lait non écrémé donc "complètement gras".

D'après les résultats d'analyses de fromages, effectuées avec nos collaborateurs, pendant plusieurs années, nous avons constaté que sous le nom ou désignation de fromage entièrement gras, on a vendu et on vend, même de nos jours (1958), sur nos marchés, toutes sortes de fromages maigres ayant très souvent moins de $10 \%$ de matière grasse dans l'extrait sec, au lieu de $45 \%$, sans aucune déclaration et au même prix que le fromage de meilleure qualité.

Il est vraiment très difficile de réaliser sous ces conditions le contrôle officiel sur chaque marché du pays, sur des milliers de petits producteurs et de vendeurs.

Il faut donc abandonner les normes compliquées et adopter une classification plus réelle. D'autre part, il faut examiner la possibilité d'adoption de dénomination de fromage "totalement gras » (Vollfettkäse), préparer du lait entier, non écrémé, et prescrire la possibilité de vente libre dans les marchés nationaux de toutes sortes de fromages obtenus du lait écrémé à la condition d'une déclaration obligatoire selon l'espèce et la teneur en matière grasse exactement désignés en pourcentage, sans dénomination " trois quart gras ", " demi gras " etc.

\section{Summary}

The most serious lack in practical application of different regulations concerning the inspection of chemical properties of cheese is the impossibility to carry out an efficient inspection of cheese applying very complicated standards in respect to their fat content in dry matter and their moisture content.

The problem is complicated by the existence of a large number of small producers who, at the same time, directly sell these products. Practically they sell cheese of all types as "whole milk cheese ", produced from whole milk which is not skimmed. However, one may find very often on sale cheese containing lesse than $10 \%$ of fat at the same price as cheese of highest quality.

Consumers are not able to evaluate the quality and value of cheese. The official inspection in our country, carried out by the Veterinary Services, based on the analytical data is practically illusory under these legislative conditions, except in cases of export. 
Therefore one should abandon complicated standards and adopt a more realistic classification. The possibility for the introduction of the term "whole milk cheese " should be explored and one should make possible to put on free sale, on local markets, all types of cheese produced from skimmed milk under condition that the type of cheese and the fat content is obligatory declared in percentage without labeling them " $3 / 4$ whole milk cheese», " $1 / 2$ whole milk, cheese» etc.

\title{
BIBLIOGRAP HIE
}

[1] W. Lethem. C. R. du XIVe Congrès International sur le lait et ses dérivés, vol. III, partie I, pp. 218-227, Rome 1956.

[2] G. Thieulin G. Ibid.pp. 265-274, Rome 1956.

[3] Réglement sur la qualité de denrées alimentaires, des conditions pour leur production et la vente (J.O. de 20-3-57, Belgrade).

[4] A. Rochaix et A. Tapernoux. Le Lait et ses Dérivés, Paris-Lyon, 1948.

[5] Recueil de Standard bulgare pour le lait et ses produits, Sofia-1956.

[6] Gunnar Aas. C. R. du XIVe Congrès International sur le lait et ses dérivés, vol. III, partie I, pp. 291-300, Rome 1956.

[7] E. Carleberg. Ibid.pp. 75-86, Rome 1956.

[8] G. Roeder. Grundzüge der Milchwirtschaft und Molkereiwesens, Hamburg-Berlin, s. 96-1954.

\section{SUPPLÉMENT TECHNIQUE}

\section{L'ACIDE LACTIQUE ET SES APPLICATIONS}

\author{
par \\ G. GENIN \\ Ingénieur E.P.C.I.
}

\section{Généralités}

La découverte de l'acide lactique remonte à environ deux cents ans et sa présence avait été reconnue à l'époque dans le lait sur, ainsi que dans les composés que l'on obtient par extraction aqueuse du tissu musculaire. Cependant, ce n'est que plus tard que l'on reconnut que, si les acides provenant de ces deux sources ne peuvent être distinguées chimiquement, ils fournissent des sels qui diffèrent par leur forme cristalline et par leurs autres propriétés physiques. C'est à la suite de cette observation qu'il fut établi que l'acide lactique peut se présenter sous deux formes constituant deux isomères optiques, la forme D et la forme L qui diffèrent par l'arrangement spatial des groupes attachés à l'un des trois atomes de carbone de l'acide. Si l'on représente la structure de ces deux acides sur un plan, on admet les deux configurations possibles suivantes : 